\title{
Object incompleteness and dynamic composition in Java-like languages ${ }^{\star}$
}

\author{
Lorenzo Bettini ${ }^{1}$, Viviana Bono ${ }^{1}$, and Betti Venneri ${ }^{2}$ \\ 1 Dipartimento di Informatica, Università di Torino, bono@di . unito.it \\ 2 Dipartimento di Sistemi e Informatica, Università di Firenze, \\ \{bettini, venneri\}@dsi.unifi.it
}

\begin{abstract}
Object composition is often advocated as a more flexible alternative to standard class inheritance since it takes place at run-time, thus permitting the behavior of objects to be specialized dynamically. In this paper we present Incomplete Featherweight Java (IFJ), an extension of Featherweight Java with incomplete objects, i.e., objects that require some missing methods which can be provided at run-time by composition with another (complete) object. Incomplete object usage is disciplined by static typing, therefore the language enjoys type safety (which implies no "message-not-understood" run-time errors).
\end{abstract}

\section{Introduction}

Standard class-based object-oriented languages rely on class inheritance, method redefinition, and dynamic binding to achieve flexibility. However, these mechanisms may not suffice for representing the dynamic behavior of objects: all the possible scenarios may not be completely predictable in advance and they are likely to change after the software application has already been developed. With the aim of forecasting all the possible evolutions of the system entities, unfortunately classes are often designed with too many responsibilities, most of which are basically not used. Furthermore, the number of subclasses tends to grow dramatically when trying to compose different functionalities into single modules. In this respect, object composition is often advocated as an alternative to class inheritance, in that it is defined at run-time and it enables dynamic object code reuse by assembling existing components [19]. Furthermore, thanks to object composition, we can exploit a programming style that builds small software components (units of reuse), that can be composed in several ways to achieve software reuse (we refer to $[27,15]$ and to the references therein for an insightful review of the limitations of inheritance).

On the other hand, object-based languages use object composition and delegation, a more flexible mechanism, to reuse code (see, e.g., the languages [28, 20, 12], and the calculi $[18,1])$. Every object has a list of parent objects: when an object cannot answer a message it forwards it to its parents until there is an instance that can process the message. However, a drawback of delegation is that run-time type errors ("messagenot-understood") can arise when no delegates are able to process the forwarded message

* This work has been partially supported by the MIUR project EOS DUE and by EU Project Software Engineering for Service-Oriented Overlay Computers (SENSORIA, contract IST-3016004-IP-09). 
[29]. In order to preserve the benefits of static type safety, several solutions have been proposed in the literature, such as design patterns [19] and language extensions integrating in class based languages more flexible mechanisms, such as, e.g., mixins [10], generic types [11], delegation (Kniesel [22] presents an overview of problems when combining delegation with static type discipline).

In this paper we present Incomplete Featherweight Java (IFJ), an extension of Featherweight Java $[21,25]$ that combines the static type discipline of class-based languages with the flexibility of object-based ones. The programmer, besides standard classes, can define incomplete classes whose instances are incomplete objects that can be composed in an object-based fashion. Hence, in our calculus it is possible: (i) to instantiate standard classes, obtaining fully-fledged objects ready to be used; (ii) to instantiate incomplete classes, obtaining incomplete objects that can be composed (by object composition) with complete objects, thus yielding new complete objects at runtime. This provides a mechanism that is similar to a sort of dynamic inheritance since it implies both substitutivity (that is, a composed object can be used where a standard object is expected) and dynamic code reuse (since composition permits supplying at run-time the missing methods with those of other objects).

Therefore, we can model some features related to dynamic object evolution: while incomplete classes separate the object invariant behavior from the variant one at compile time, at run-time object composition customizes the unpredictable behavior based on dynamic conditions (for instance, the object state) in a type safe way. In particular, some behavior that was not foreseen when the class hierarchy was implemented might be supplied dynamically by making use of already existing objects, thus generating an unanticipated reuse of code and a sharing relationship of components.

Concerning the theory of incomplete objects, our main inspiration comes from [4]; however, while that calculus builds on top of the lambda calculus, here we aim at proving how object composition can fit within the basic principles of Java-like languages. This leads to some key design choices. Firstly, instead of employing structural subtyping as in [4], we keep the nominal subtyping mechanism that is typical of mainstream languages like Java and $\mathrm{C}++$. This feature allows us to define an extension that is conservative with respect to the core Java, since it does not affect those parts of the programs that do not use incomplete objects. Furthermore, incomplete classes can rely on standard class inheritance to reuse code of parent classes (although this kind of inheritance does not imply subtyping). Thus incomplete objects provide two forms of code reuse: vertical (i.e., the code reuse achieved via standard class inheritance) and horizontal (i.e., the one achieved via object composition). Finally, in order to enhance run-time flexibility in composing objects we implicitly use structural subtyping during composition: an incomplete object can be composed with any object providing all the requested methods (the signatures must match) independently of the classes of these objects. Then the language extension we propose is not a simple automatic implementation of the object composition that one might implement manually. In fact, any object providing the required methods can be used in object completion, no matter what its class is. In case of a manual implementation, instead, the object should be stored in a class field thus forcing it to belong to a specific class hierarchy (see Section 2.2). 
Concerning the semantics of method invocation on objects that are obtained by composition, our calculus implements consultation but not delegation (see Section 2.4), though in Section 5 we sketch an extension to delegation, the subject of future work. Let us note that, in the literature (e.g., [19]), the term delegation, originally introduced by Lieberman [23], is given different interpretations and it is often confused with the term consultation (sometimes simply called forwarding). In both cases an object $A$ has a reference to an object $B$. However, when $A$ forwards to $B$ the execution of a message $m$, two different bindings of the implicit parameter this can be adopted for the execution of the body of $m$ : with delegation, this is bound to the sender $(A)$ thus, if in the body of the method $m$ (defined in $B$ ) there is a call to a method $n$, then also this call will be executed binding this to $A$; with consultation, during the execution of the body the implicit parameter is always bound to the receiver $B$.

Our proposal also deals with possible additional methods (hidden by subsumption) that might rise the "width subtyping versus method addition" problem that is well known in the object-based setting (see, e.g., [18]). We solve this issue by representing objects as lists of subobjects in such a way that we can explicitly deal with the "scope" of a method invocation; we believe this solution is much more implementation oriented than the dictionaries of [26] and simpler than the one of [4].

Summarizing, our main intention is to have a language with a tradeoff between the dynamic flexibility that is typical of object-based languages and the static discipline of class-based languages. Objects are then still instances of classes (possibly incomplete classes) and they are still disciplined by the nominal subtyping, but they are also prototypes that can be used, via the object composition, to create new objects at run-time, while ensuring statically that the composition is type safe.

There are, in fact, settings in which it is important to exploit software units that are as simple as possible to facilitate reuse and, at the same time, flexible enough to enable dynamic reconfiguration and re-purposing. An example of such a scenario is the one of learning objects in the context of eLearning environments [9]. Standard learning objects are characterized by the lack of sufficient support for adaptive behavior. Therefore, a new view and implementation of adaptive learning objects may be obtained by exploiting incomplete objects that can be safely and independently used in different compositions. Foremost, these objects compositions are performed dynamically, i.e., at interaction time, since they can depend on information about the state or the history of the learner. Thus incomplete learning objects can satisfy basic software engineering principles, while providing the required pedagogical richness.

The calculus presented in this paper has a reduced set of features since we wanted to investigate the problem of safe and dynamic object composition in a core formal framework and to have a kernel language providing the basis for future enhancements and experiments with object compositions (some possible extensions are sketched in Section 5). We also wanted this core basis to be sound; in fact, our language enjoys the type safety property (see Section 3) which guarantees that in a well-typed program no "message-not-understood" can occur at run-time (this will also exclude possible runtime accesses to methods of an incomplete object). 


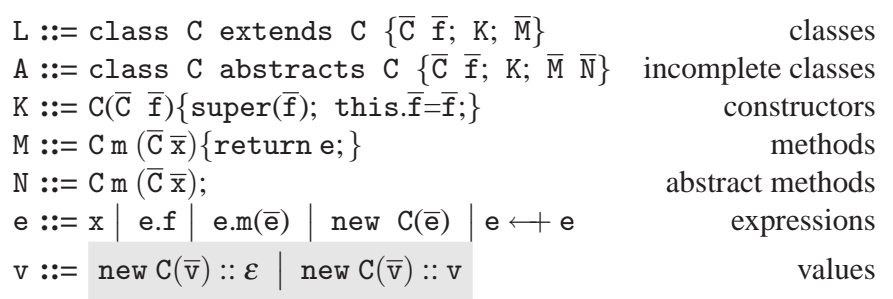

Fig. 1. IFJ syntax; run-time syntax appears shaded.

\section{Incomplete Featherweight Java}

In this section we present syntax, typing and operational semantics of our proposal, the core language IFJ (Incomplete Featherweight Java), which is an extension of FJ (Featherweight Java) with incomplete objects. FJ [21, 25] is a lightweight version of Java, which focuses on a few basic features: mutually recursive class definitions, inheritance, object creation, method invocation, method recursion through this, subtyping and field access ${ }^{2}$. Thus, the minimal syntax, typing and semantics make the type safety proof simple and compact, in such a way that FJ is a handy tool for studying the consequences of extensions and variations with respect to Java ("FJ's main application is modeling extensions of Java", [25], pag. 248). Although we assume the reader is familiar with FJ, we will briefly comment on the FJ part and then we will focus on the novel aspects introduced by IFJ.

The abstract syntax of IFJ constructs is given in Figure 1 and it is just the same as FJ extended with incomplete classes, abstract methods and object composition (and some run-time expressions that are not written by the programmer, but are produced by the semantics, that we will discuss later, Section 2.4). The metavariables B, C, D and $\mathrm{E}$ range over class names (both concrete and incomplete); $\mathrm{M}$ ranges over (standard) method definitions and $N$ ranges over (abstract) method signatures; $f$ and $g$ range over attribute names; $\mathrm{x}$ ranges over method parameter names; e and d range over expressions and $\mathrm{v}$ and $\mathrm{u}$ range over values. As in FJ, we will use the overline notation for possibly empty sequences (e.g., " $\bar{e}$ " is a shorthand for a possibly empty sequence " $e_{1}, \ldots, e_{n}$ "). We abbreviate pair of sequences in a similar way, e.g., $\bar{C} \bar{f}$ stands for $C_{1} f_{1}, \ldots, C_{n} f_{n}$. The empty sequence is denoted by $\bullet$. Following FJ, we assume that the set of variables includes the special variable this (implicitly bound in any method declaration), which cannot be used as the name of a method's formal parameter (this restriction is imposed by the typing rules). Note that since we treat this in method bodies as an ordinary variable, no special syntax for it is required.

A class declaration class $C$ extends $D\{\bar{C} \overline{\mathrm{f}} ; \mathrm{K} ; \overline{\mathrm{M}}\}$ consists of its name $\mathrm{C}$, its superclass D (which must always be specified, even if it is Object), a list of field names $\bar{C} \bar{f}$ with their types, the constructor $K$, and a list of method definitions $\bar{M}$. The fields of $\mathrm{C}$ are added to the ones declared by $\mathrm{D}$ and its superclasses and are assumed to have distinct names. The constructor declaration shows how to initialize all these fields

\footnotetext{
${ }^{2}$ FJ also includes up and down casts; however, since these features are completely orthogonal to our extension, they are omitted in IFJ.
} 
with the received values. A method definition $M$ specifies the name, the signature and the body of a method; a body is a single return statement since FJ is a functional core of Java. In the following, we will write $\mathrm{m} \notin \overline{\mathrm{M}}$ to mean that the method definition of the name $m$ is not included in $\overline{\mathrm{M}}$. The same convention will be used for method signatures $\overline{\mathrm{N}}$.

An incomplete class declaration class $C$ abstracts $D\{\bar{C} \overline{\mathrm{f}} ; \mathrm{K} ; \overline{\mathrm{M}} \overline{\mathrm{N}}\}$ inherits from a standard (or incomplete) class and, apart from adding new fields and adding/overriding methods, it can declare some methods as "incomplete" (we will call these methods also "abstract" or "expected"). Note that, on the other hand, standard classes cannot inherit from incomplete classes (this is checked by typing, Section 2.3). The main idea of our language, is that an incomplete class can be instantiated, leading to incomplete objects. Method invocation and field selection cannot be performed on incomplete objects ${ }^{3}$.

An incomplete object expression $\mathrm{e}_{1}$ can be composed at run-time with a complete object expression $\mathrm{e}_{2}$; this operation, denoted by $\mathrm{e}_{1} \longleftarrow \mathrm{e}_{2}$, is called object composition. The key idea is that $e_{1}$ can be composed with a complete object $e_{2}$ that provides all the requested methods, independently from the class of $e_{2}$ (of course, the method signatures must match). Then, in $\mathrm{e}_{1} \leftarrow \mathrm{e}_{2}, \mathrm{e}_{1}$ must be an incomplete object and $\mathrm{e}_{2}$ must be a complete object expression (these requirements are checked by the type system); indeed, $e_{2}$ can be, in turn, the result of another object composition. The object expression $\mathrm{e}_{1} \leftarrow \mathrm{e}_{2}$ represents a brand new (complete) object that consists of the sub-object expressions $e_{1}$ and $e_{2}$; in particular, the objects of these sub-expressions are not modified during the composition. In Section 4 we will get into more details about this point, and about how the language can be smoothly extended to the imperative setting. This also highlights the roles of incomplete and complete objects as re-usable building blocks for new objects at run-time, while retaining their identity and state. This is also another main difference with respect to [4], where incomplete objects are modified during the object composition (and thus they could not be re-used in further compositions).

We note that in this basic version of the language we do not allow object composition operations leading to incomplete objects, i.e., incomplete objects can only be fully completed. However, for instance, object compositions of the shape $\left(e_{1} \leftarrow e_{2}\right) \longleftarrow e_{3}$, where $e_{2}$ is incomplete in the methods provided by $e_{3}$, can be obtained as $e_{1} \longleftarrow\left(e_{2} \longleftarrow\right.$ $e_{3}$ ) in IFJ. Furthermore, we prohibit the object composition between two complete objects: the semantics would not be clear and could lead to unexpected behaviors at runtime (for instance, a further composition of an object o with a complete object might lead to an accidental method overriding, because there might be a method in $\circ$ with the same name and the same signature, but with a completely different semantics, of a method already present in the complete object). It is part of our main goal to keep the discipline that is typical of statically typed class based languages where some crucial intentions (e.g., when a method can be redefined) must be written explicitly by the programmer: during object composition, the requested methods must be explicitly declared in the incomplete classes.

Finally, values, denoted by $\mathrm{v}$ and $\mathrm{u}$, are fully evaluated object creation terms. However, the object representation of IFJ is different from FJ in that fully evaluated objects

\footnotetext{
${ }^{3}$ Actually, field selection might be safely performed on incomplete objects but would make a little sense.
} 


$$
\begin{gathered}
\mathrm{T}<: \mathrm{T} \quad \frac{\mathrm{T}_{1}<: \mathrm{T}_{2} \quad \mathrm{~T}_{2}<: \mathrm{T}_{3}}{\mathrm{~T}_{1}<: \mathrm{T}_{3}} \quad \frac{\text { class C extends } \mathrm{D}\{\ldots\}}{\mathrm{C}<: \mathrm{D}} \\
\frac{\text { class C abstracts } \mathrm{D}\{\ldots\} \quad \text { class D extends } \mathrm{E}\{\ldots\}}{\langle\mathrm{C}\rangle<: \mathrm{D}} \\
\frac{\text { class C abstracts } \mathrm{D}\{\ldots\} \quad \text { class D abstracts } \mathrm{E}\{\ldots\}}{\langle\mathrm{C}\rangle<:\langle\mathrm{D}\rangle}
\end{gathered}
$$

Fig. 2. Subtyping rules.

can be also compositions of many objects. Thus, objects are represented as lists of terms new $\mathrm{C}(\overline{\mathrm{v}})$ (i.e., expressions that are passed to the constructor are values too). For instance, new $\mathrm{C}(\overline{\mathrm{v}})::$ new $\mathrm{D}(\overline{\mathrm{u}}):: \varepsilon$ represents the composition of the incomplete object of class $C$ with a standard complete object of class D ( $\varepsilon$ denotes the empty list). This run-time representation of objects will be further explained in Section 2.4.

As in FJ, a class table $C T$ is a mapping from class names to class declarations. Then a program is a pair $(C T, \mathrm{e})$ of a class table (containing all the class definitions of the program) and an expression e (the program's main entry point). The class Object has no members and its declaration does not appear in $C T$. We assume that $C T$ satisfies some usual sanity conditions: $(i) C T(\mathrm{C})=$ class $\mathrm{C} \ldots$ for every $\mathrm{C} \in \operatorname{dom}(C T)$ (ii) for every class name $\mathrm{C}$ (except Object) appearing anywhere in $C T$, we have $\mathrm{C} \in \operatorname{dom}(C T)$; (iii) there are no cycles in the transitive closure of the extends relation. Thus, in the following, instead of writing $C T(\mathrm{C})=$ class $\ldots$ we will simply write class $\mathrm{C} \ldots$.

\subsection{Subtyping}

In the type system we will need to distinguish between the type of an incomplete object and the type of a composed object (i.e., an incomplete object that has been composed with a complete object). If $\mathrm{C}$ is the class name of an incomplete object, then $\langle\mathrm{C}\rangle$ is the type of an incomplete object of class $\mathrm{C}$ that has been composed. To treat complete and incomplete objects uniformly, we will use $\mathrm{T}$ to refer both to $\mathrm{C}$ and $\langle\mathrm{C}\rangle$. However, types of the shape $\langle\mathrm{C}\rangle$ are only used by the type system for keeping track of objects that are created via object composition. Indeed the programmer cannot write $\langle C\rangle$ explicitly, i.e., $\mathrm{T}$ cannot be used in arrow types nor for declaring method parameters; this is consistent with Java-like languages' philosophy where the class names are the only types that can be mentioned in the program (apart from basic types).

The subtype relation $<$ : (defined for any class table $C T$ ) on classes (types) is induced by the standard subclass relation extended in order to relate incomplete objects (Figure 2). First of all, let us consider an incomplete class class C abstracts D $\{\ldots\}$; if $\mathrm{D}$ is a standard class, since $\mathrm{C}$ can make some methods of $\mathrm{D}$ incomplete, then it is obvious that an incomplete object of class $C$ cannot be used in place of an object of class D. Thus, abstracts implements subclassing without subtyping. Instead, when the incomplete object is composed with a complete object (providing all the methods requested by $\mathrm{C})$, then its type is $\langle\mathrm{C}\rangle$, and it can be used in place of an object of class $\mathrm{D}$ (see third rule). Since, as said above, we do not permit object completion on a complete 
object, then a complete object can never be used in place of an incomplete one. Introducing subtyping between incomplete objects would require checking that the subtype does not have more incomplete methods than the supertype (contra-variance on requirements). To keep the presentation simple, however, in this first version we are not considering subtyping on incomplete objects (see Section 5). Instead, subtyping holds on their completed versions (last rule).

\subsection{Programming Examples}

In this section, we show how incomplete objects, and object composition, can be used to implement some recurrent programming scenarios. For simplicity, we will use here the full Java syntax (consider all methods as public) and we will denote object completion operation with $<-$.

We consider a scenario where it is useful to add some functionality to existing objects. Let us consider the development of an application that uses widgets such as graphical buttons, menus, and keyboard shortcuts. These widgets are usually associated to an event listener (e.g., a callback function), that is invoked when the user sends an event to that specific widget (e.g., one clicks the button with the mouse or chooses a menu item).

The design pattern command [19] is useful for implementing these scenarios, since it permits parametrization of widgets over the event handlers, and the same event handler can be reused for similar widgets (e.g., the handler for the event "save file" can be associated with a button, a menu item, or a keyboard shortcut). Thus, they delegate to this object the actual implementation of the action semantics, while the action widget itself abstracts from it. This decouples the action visual representation from the action controller implementation.

We can implement directly this scenario with incomplete objects, as shown in Listing 1: the class Action and SaveActionDelegate are standard Java classes (note that they're not related). The former is a generic implementation of an action, and the latter implements the code for saving a file. We then have three incomplete classes implementing a button, a menu item, and a keyboard accelerator; note that these classes inherit from Action and make the method run incomplete and override the method display. We also assume a class Frame representing an application frame where we can set keyboard accelerators, menu items, and toolbar buttons. An instance of class Button is an incomplete object (it requires the method run) and, as such, we cannot pass it to addToToolbar, since Button $\nless$ : Action (subclassing without subtyping). However, once we composed such instance (through object completion operation, <-) with an instance of SaveActionDelegate, then we have a completed object (of type $\langle$ Button〉) that can be passed to addToToolbar (since $\langle$ Button $\rangle<$ : Action). Note that we compose Button with an instance of SaveActionDelegate which provides the requested method run, although SaveActionDelegate is not related to Action. Furthermore, we can use the same instance of SaveActionDelegate for the other incomplete objects.

We conclude this section by discussing some possible manual implementations in Java of this scenario, showing that our proposal is not simply syntactic sugar. With standard Java features, one could write the Button class with a field, say deleg, on 


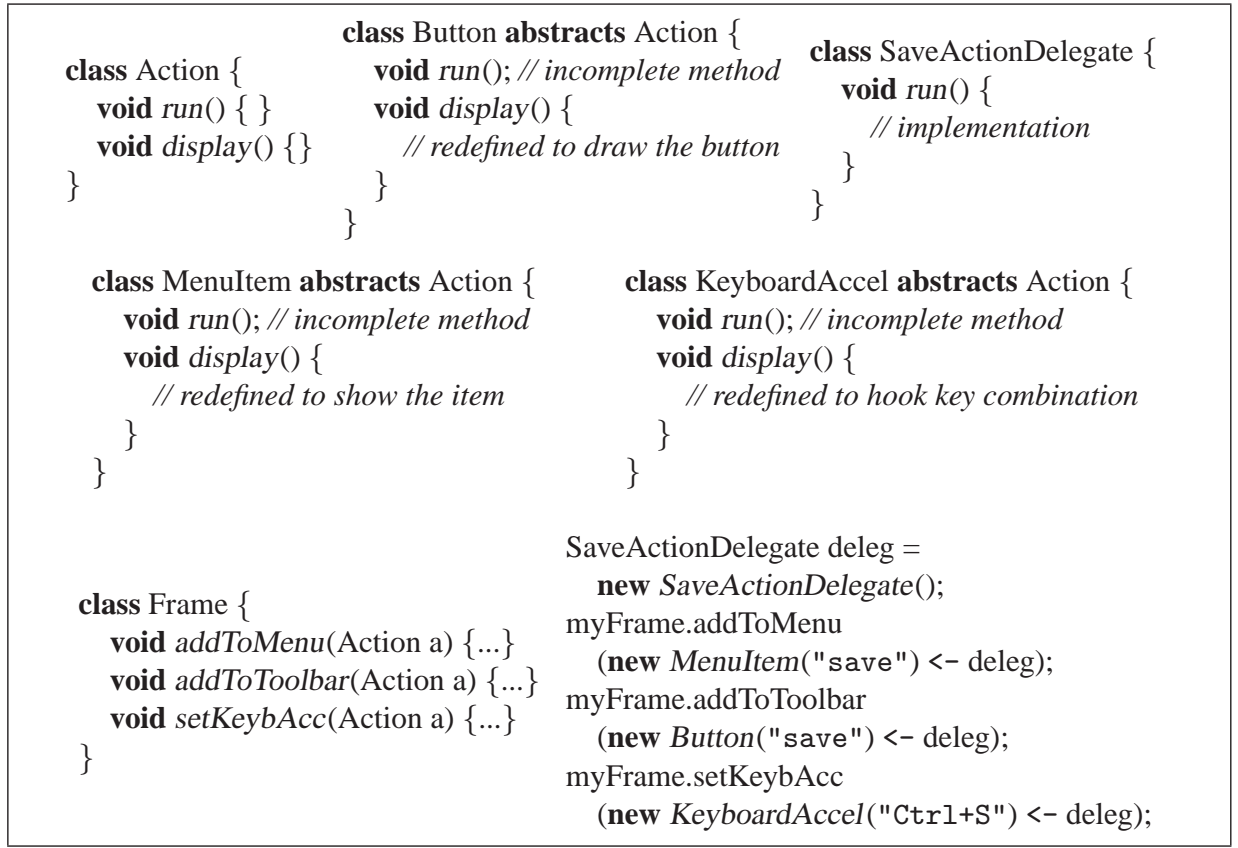

Listing 1: The implementation of action and action delegates with incomplete objects and object completion

which we call the method run. This approach requires deleg to be declared with a class or interface that provides such method, say Runnable. However, this solution would not be as flexible as our incomplete objects, since one can then assign to deleg only objects belonging to the Runnable hierarchy.

On the other hand, if one wanted to keep the flexibility, he should declare deleg of type Object, and then call the method run by using Java Reflection APIs, (e.g., getMethod); however, this solution is not type safe, since exceptions can be thrown at run-time due to missing methods.

\subsection{Typing}

In order to define the typing rules and the lookup functions, we extend the sequence notation also to method definitions:

$$
\overline{\mathrm{C} m(\overline{\mathrm{C}} \overline{\mathrm{x}})\{\text { return e; }\}}
$$

represents a sequence of method definitions:

$$
\mathrm{C}_{1} \mathrm{~m}_{1}\left(\overline{\mathrm{C}}_{1} \overline{\mathrm{x}}\right)\left\{\text { return } \mathrm{e}_{1} ;\right\} \ldots \mathrm{C}_{n} \mathrm{~m}_{n}\left(\overline{\mathrm{C}}_{n} \overline{\mathrm{x}}\right)\left\{\text { return } \mathrm{e}_{n} ;\right\}
$$

The signatures of the above method definitions will be denoted, in a compact form,

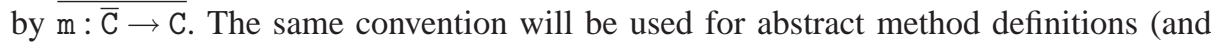


their corresponding signatures). To lighten the notation, in the following, we will assume a fixed class table $C T$ and then $<:$ is the subtype relation induced by $C T$. We will write $\overline{\mathrm{C}}<: \overline{\mathrm{D}}$ as a shorthand for $\mathrm{C}_{1}<: \mathrm{D}_{1} \wedge \ldots \wedge \mathrm{C}_{n}<: \mathrm{D}_{n}$.

$$
\begin{aligned}
& \text { fields }(\mathrm{Object})=\bullet \\
& \text { class C extends D }\{\overline{\mathrm{C}} \overline{\mathrm{f}} ; \mathrm{K} ; \overline{\mathrm{M}}\} \quad \text { fields }(\mathrm{D})=\overline{\mathrm{D}} \overline{\mathrm{g}} \\
& \text { fields }(\mathrm{C})=\overline{\mathrm{D}} \overline{\mathrm{g}}, \overline{\mathrm{C}} \overline{\mathrm{f}} \\
& \frac{\text { class C abstracts D }\{\overline{\mathrm{C}} \overline{\mathrm{f}} ; \mathrm{K} ; \overline{\mathrm{M}} \overline{\mathrm{N}}\} \quad \text { fields }(\mathrm{D})=\overline{\mathrm{D}} \overline{\mathrm{g}}}{\text { fields }(\mathrm{C})=\overline{\mathrm{D}} \overline{\mathrm{g}}, \overline{\mathrm{C}} \overline{\mathrm{f}}} \\
& \text { fields }(\langle\mathrm{C}\rangle)=\text { fields }(\mathrm{C}) \\
& \text { class C extends D }\{\overline{\mathrm{C}} \overline{\mathrm{f}} ; \mathrm{K} ; \overline{\mathrm{M}}\} \\
& \frac{\overline{\mathrm{M}}=\overline{\mathrm{Bm}(\overline{\mathrm{B}} \overline{\mathrm{x}})\{\text { return } \mathrm{e} ;\}} \quad \operatorname{sign}(\mathrm{D})=\langle\mathrm{S}, \emptyset\rangle}{\operatorname{sign}(\mathrm{C})=\langle\{\overline{\mathrm{m}: \overline{\mathrm{B}} \rightarrow \mathrm{B}}\} \cup \mathrm{S}, \emptyset\rangle} \\
& \text { class C abstracts } \mathrm{D}\{\overline{\mathrm{C}} \overline{\mathrm{f}} ; \mathrm{K} ; \overline{\mathrm{M}} \overline{\mathrm{N}}\} \quad \operatorname{sign}(\mathrm{D})=\left\langle\mathrm{S}_{1}, \mathrm{~S}_{2}\right\rangle \\
& \overline{\mathrm{M}}=\overline{\mathrm{Bm}(\overline{\mathrm{B}} \overline{\mathrm{x}})\{\text { return e }\}} \quad \overline{\mathrm{N}}=\overline{\mathrm{En}(\overline{\mathrm{E}} \overline{\mathrm{x}})} ; \\
& \overline{\operatorname{sign}(\mathrm{C})}=\left\langle\{\overline{\mathrm{m}: \overline{\mathrm{B}} \rightarrow \mathrm{B}}\} \cup\left(\mathrm{S}_{1}-\{\overline{\mathrm{n}: \overline{\mathrm{E}} \rightarrow \mathrm{E}}\}\right),\{\overline{\mathrm{n}: \overline{\mathrm{E}} \rightarrow \mathrm{E}}\} \cup\left(\mathrm{S}_{2}-\{\overline{\mathrm{m}: \overline{\mathrm{B}} \rightarrow \mathrm{B}}\}\right)\right\rangle \\
& \frac{\operatorname{sign}(\mathrm{C})=\left\langle\mathrm{S}_{1}, \mathrm{~S}_{2}\right\rangle}{\operatorname{sign}(\langle\mathrm{C}\rangle)=\left\langle\mathrm{S}_{1} \cup \mathrm{S}_{2}, \emptyset\right\rangle} \\
& \frac{\operatorname{sign}(\mathrm{T})=\left\langle\mathrm{S}_{1}, \mathrm{~S}_{2}\right\rangle \quad \mathrm{m}: \overline{\mathrm{B}} \rightarrow \mathrm{B} \in \mathrm{S}_{1} \cup \mathrm{S}_{2}}{\text { mtype }(\mathrm{m}, \mathrm{T})=\overline{\mathrm{B}} \rightarrow \mathrm{B}} \\
& \frac{\text { class C extends D }\{\overline{\mathrm{C}} \overline{\mathrm{f}} ; \mathrm{K} ; \overline{\mathrm{M}}\} \quad \mathrm{B} \mathrm{m}(\overline{\mathrm{B}} \overline{\mathrm{x}})\{\text { return } \mathrm{e} ;\} \in \overline{\mathrm{M}}}{\operatorname{mbody}(\mathrm{m}, \mathrm{C})=\overline{\mathrm{x}} . \mathrm{e}} \\
& \frac{\text { class C extends D }\{\overline{\mathrm{C}} \overline{\mathrm{f}} ; \mathrm{K} ; \overline{\mathrm{M}}\} \quad \mathrm{m} \notin \overline{\mathrm{M}}}{\operatorname{mbody}(\mathrm{m}, \mathrm{C})=\operatorname{mbody}(\mathrm{m}, \mathrm{D})} \\
& \text { class C abstracts D }\{\overline{\mathrm{C}} \overline{\mathrm{f}} ; \mathrm{K} ; \overline{\mathrm{M}} \overline{\mathrm{N}}\} \quad \mathrm{Bm}(\overline{\mathrm{B}} \overline{\mathrm{x}})\{\text { return e; }\} \in \overline{\mathrm{M}} \\
& \operatorname{mbody}(\mathrm{m}, \mathrm{C})=\overline{\mathrm{x}} . \mathrm{e} \\
& \frac{\text { class C abstracts D }\{\overline{\mathrm{C}} \overline{\mathrm{f}} ; \mathrm{K} ; \overline{\mathrm{M}} \overline{\mathrm{N}}\} \quad \mathrm{m} \notin \overline{\mathrm{M}} \quad \mathrm{m} \notin \overline{\mathrm{N}}}{\operatorname{mbody}(\mathrm{m}, \mathrm{C})=\operatorname{mbody}(\mathrm{m}, \mathrm{D})} \\
& \text { class C abstracts D }\{\overline{\mathrm{C}} \overline{\mathrm{f}} ; \mathrm{K} ; \overline{\mathrm{M}} \overline{\mathrm{N}}\} \quad \mathrm{B} \mathrm{m}(\overline{\mathrm{B}} \overline{\mathrm{x}}) ; \in \overline{\mathrm{N}} \\
& \operatorname{mbody}(\mathrm{m}, \mathrm{C})=\bullet
\end{aligned}
$$

Fig. 3. Lookup functions. 
We define auxiliary functions (see Figure 3) to lookup fields and method from $C T$; these functions are used in the typing rules and in the operational semantics.

A signature set, denoted by $\mathrm{S}$ is a set of method signatures of the shape $\mathrm{m}: \overline{\mathrm{C}} \rightarrow \mathrm{C}$. The signature of a class $\mathrm{C}$, denoted by $\operatorname{sign}(\mathrm{C})$, is a pair of signature sets $\left\langle\mathrm{S}_{1}, \mathrm{~S}_{2}\right\rangle$, where the first set is the signature set of the defined methods and the second set is the signature set of the abstract methods. Of course, for standard classes, the second set will be empty.

The lookup function fields $(\mathrm{C})$ returns the sequence of the field names, together with the corresponding types, for all the fields declared in $\mathrm{C}$ and in its superclasses. The mtype $(\mathrm{m}, \mathrm{C})$ lookup function (where $\mathrm{m}$ is the method name we are looking for, and $\mathrm{C}$ is the class where we are performing the lookup) differs from the one of FJ in that it relies on the new lookup function sign; the lookup function sign returns the signature of a class by inspecting the signature of its type. In particular, since the superclass D of an incomplete class $\mathrm{C}$ can be in turn an incomplete class, the methods that are complete are those defined in $C$ and those defined in $D$ that are not made incomplete by $C$ (i.e., $\left.\{\overline{\mathrm{m}: \overline{\mathrm{B}} \rightarrow \mathrm{B}}\} \cup\left(\mathrm{S}_{1}-\{\overline{\mathrm{n}: \overline{\mathrm{E}} \rightarrow \mathrm{E}}\}\right)\right)$; conversely, the incomplete methods are the abstract methods of $C$ and those of $D$ that are not (re)defined in $C$ (i.e., $\{\overline{\mathrm{n}: \overline{\mathrm{E}} \rightarrow \mathrm{E}}\} \cup\left(\mathrm{S}_{2}-\right.$ $\{\overline{\mathrm{m}: \overline{\mathrm{B}} \rightarrow \mathrm{B}}\})$ ). Moreover, for a composed object of type $\langle\mathrm{C}\rangle$ it returns a signature where the first element is the union of the signature sets of its class and the second element is made empty; this reflects the fact that now all the methods of the object are concrete. Since we introduced this lookup function, the definition of mtype is straightforward (w.r.t. the one of FJ [21]). The lookup function for method bodies, mbody, is basically the same definition of FJ extended to incomplete classes (note that it returns an empty element $\bullet$ for abstract methods).

A type judgment of the form $\Gamma \vdash \mathrm{e}: \mathrm{T}$ states that "e has type $\mathrm{T}$ in the type environment $\Gamma$ ". A type environment is a finite mapping from variables (including this) to types, written $\overline{\mathrm{x}}: \overline{\mathrm{C}}$. Again, we use the sequence notation for abbreviating $\Gamma \vdash \mathrm{e}_{1}: \mathrm{T}_{1}, \ldots, \Gamma \vdash \mathrm{e}_{n}: \mathrm{T}_{n}$ to $\Gamma \vdash \overline{\mathrm{e}}: \overline{\mathrm{T}}$.

Typing rules (Figure 4) are adapted from those of FJ in order to handle incomplete objects and object composition. In particular, field selection and method selection are allowed only on objects of concrete types, where a concrete type is either a standard class $\mathrm{C}$ or $\langle\mathrm{C}\rangle$. The key rule (T-COMP) for dealing with object composition is introduced. It checks that the left expression is actually an incomplete object $\left(\mathrm{S}_{2} \neq \emptyset\right)$, and that the right one is a complete object that provides all the methods needed by the incomplete object. Note that the final type is the concrete type based on the original class of the incomplete object. This rule also shows that the typing of $\longleftarrow$ is structural, which is a key feature of the system, since it enhances the flexibility of object composition.

Also typing rules for methods and classes of FJ are adapted to deal with incomplete classes (note the use of the override predicate of [25] to check that the signature of a method is preserved by method overriding). Moreover, (T-CLASS) checks that a concrete class extends another concrete class and (T-ACLASS) checks that also the signatures of incomplete methods satisfy the override predicate. Typing rules for run-time expressions are in Figure 5; note that the type of a composed object is taken from the head of the list, consistently with the typing rule for object completion. 
concrete predicate

$$
\frac{\operatorname{sign}(\mathrm{C})=\langle\mathrm{S}, \emptyset\rangle}{\operatorname{concrete}(\mathrm{C})} \quad \operatorname{concrete}(\langle\mathrm{C}\rangle)
$$

Expression typing

$$
\begin{aligned}
& \Gamma \vdash \mathrm{x}: \Gamma(\mathrm{x}) \\
& \frac{\Gamma \vdash \mathrm{e}: \mathrm{T} \quad \text { fields }(\mathrm{T})=\overline{\mathrm{C}} \overline{\mathrm{f}} \quad \operatorname{concrete}(\mathrm{T})}{\Gamma \vdash \mathrm{e} . \mathrm{f}_{i}: \mathrm{C}_{i}} \\
& \frac{\Gamma \vdash \mathrm{e}: \mathrm{T} \quad \Gamma \vdash \overline{\mathrm{e}}: \overline{\mathrm{T}} \quad \text { mtype }(\mathrm{m}, \mathrm{T})=\overline{\mathrm{B}} \rightarrow \mathrm{B} \quad \overline{\mathrm{T}}<: \overline{\mathrm{B}} \quad \text { concrete }(\mathrm{T})}{\Gamma \vdash \mathrm{e} \cdot \mathrm{m}(\overline{\mathrm{e}}): \mathrm{B}} \\
& \frac{\text { fields }(\mathrm{C})=\overline{\mathrm{D}} \overline{\mathrm{f}} \quad \Gamma \vdash \overline{\mathrm{e}}: \overline{\mathrm{T}} \quad \overline{\mathrm{T}}<: \overline{\mathrm{D}}}{\Gamma \vdash \text { new } \mathrm{C}(\overline{\mathrm{e}}): \mathrm{C}} \\
& \Gamma \vdash \mathrm{e}_{1}: \mathrm{C} \quad \operatorname{sign}(\mathrm{C})=\left\langle\mathrm{S}_{1}, \mathrm{~S}_{2}\right\rangle \quad \mathrm{S}_{2} \neq \emptyset \\
& \frac{\Gamma \vdash \mathrm{e}_{2}: \mathrm{T} \quad \operatorname{sign}(\mathrm{T})=\left\langle\mathrm{S}_{1}^{\prime}, \emptyset\right\rangle \quad \mathrm{S}_{2} \subseteq \mathrm{S}_{1}^{\prime}}{\Gamma \vdash \mathrm{e}_{1} \longleftarrow \mathrm{e}_{2}:\langle\mathrm{C}\rangle}
\end{aligned}
$$

override predicate

$$
\frac{\text { mtype }(\mathrm{m}, \mathrm{D})=\overline{\mathrm{C}} \rightarrow \mathrm{C} \text { implies } \overline{\mathrm{C}}=\overline{\mathrm{B}} \text { and } \mathrm{C}=\mathrm{B}}{\text { override }(\mathrm{m}, \mathrm{D}, \overline{\mathrm{B}} \rightarrow \mathrm{B})}
$$

\section{Method and Class typing}

$$
\begin{aligned}
& \overline{\mathrm{x}}: \overline{\mathrm{B}} \text {, this }: \mathrm{C} \vdash \mathrm{e}: \mathrm{T} \quad \mathrm{T}<: \mathrm{B} \\
& \text { class } \mathrm{C} \text { extends } \mathrm{D}\{\overline{\mathrm{C}} \overline{\mathrm{f}} ; \mathrm{K} ; \overline{\mathrm{M}}\} \quad \operatorname{override}(\mathrm{m}, \mathrm{D}, \overline{\mathrm{B}} \rightarrow \mathrm{B}) \\
& \text { B m }(\bar{B} \bar{x})\{\text { return e; }\} \text { OK IN C } \\
& \overline{\mathrm{x}}: \overline{\mathrm{B}} \text {, this }: \mathrm{C} \vdash \mathrm{e}: \mathrm{T} \quad \mathrm{T}<: \mathrm{B} \\
& \frac{\text { class C abstracts } D\{\overline{\mathrm{C}} \overline{\mathrm{f}} ; \mathrm{K} ; \overline{\mathrm{M}} \overline{\mathrm{N}}\} \quad \text { override }(\mathrm{m}, \mathrm{D}, \overline{\mathrm{B}} \rightarrow \mathrm{B})}{\mathrm{B} \mathrm{m}(\overline{\mathrm{B}} \overline{\mathrm{x}})\{\text { return e; }\} \text { OK IN C }} \\
& \frac{\text { class C abstracts } D\{\overline{\mathrm{C}} \overline{\mathrm{f}} ; \mathrm{K} ; \overline{\mathrm{M}} \overline{\mathrm{N}}\} \quad \operatorname{override}(\mathrm{m}, \mathrm{D}, \overline{\mathrm{B}} \rightarrow \mathrm{B})}{\mathrm{Bm}(\overline{\mathrm{B}} \overline{\mathrm{x}}) ; \mathrm{OK} \text { IN } \mathrm{C}} \\
& \mathrm{K}=\mathrm{C}(\overline{\mathrm{D}} \overline{\mathrm{g}}, \overline{\mathrm{C}} \overline{\mathrm{f}})\{\operatorname{super}(\overline{\mathrm{g}}) ; \text { this. } \overline{\mathrm{f}}=\overline{\mathrm{f}} ;\} \\
& \frac{\text { fields }(\mathrm{D})=\overline{\mathrm{D}} \overline{\mathrm{g}} \quad \overline{\mathrm{M}} \mathrm{OK} \text { IN C } \quad \text { concrete }(\mathrm{D})}{\text { class C extends D }\{\overline{\mathrm{C}} \overline{\mathrm{f}} ; \mathrm{K} ; \overline{\mathrm{M}}\} \mathrm{OK}} \\
& \mathrm{K}=\mathrm{C}(\overline{\mathrm{D}} \overline{\mathrm{g}}, \overline{\mathrm{C}} \overline{\mathrm{f}})\{\operatorname{super}(\overline{\mathrm{g}}) ; \text { this. } \overline{\mathrm{f}}=\overline{\mathrm{f}} ;\} \\
& \frac{\operatorname{fields}(\mathrm{D})=\overline{\mathrm{D}} \overline{\mathrm{g}} \quad \overline{\mathrm{M}} \mathrm{OK} \text { IN C } \quad \overline{\mathrm{N}} \mathrm{OK} \text { IN C }}{\text { class C abstracts D }\{\overline{\mathrm{C}} \overline{\mathrm{f}} ; \mathrm{K} ; \overline{\mathrm{M}} \overline{\mathrm{N}}\} \text { OK }}
\end{aligned}
$$

(T-Method)

Fig. 4. Typing rules. 


$$
\frac{\Gamma \vdash \text { new C }(\overline{\mathrm{v}}): \mathrm{C}}{\Gamma \vdash \text { new } \mathrm{C}(\overline{\mathrm{v}}):: \varepsilon: \mathrm{C}}(\mathrm{T}-\text { ListH }) \quad \frac{\Gamma \vdash \text { new } \mathrm{C}(\overline{\mathrm{v}}): \mathrm{C} \quad \Gamma \vdash \mathrm{v}: \mathrm{T}}{\Gamma \vdash \text { new } \mathrm{C}(\overline{\mathrm{v}}):: \mathrm{v}:\langle\mathrm{C}\rangle} \text { (T-List) }
$$

Fig. 5. Run-time expression typing

\section{Reduction}

$$
\begin{array}{cr}
\text { new } \mathrm{C}(\overline{\mathrm{v}}) \longrightarrow \operatorname{new} \mathrm{C}(\overline{\mathrm{v}}):: \varepsilon & \text { (R-NEw) } \\
\text { new } \mathrm{C}(\overline{\mathrm{v}}):: \varepsilon \longleftarrow \mathrm{v} \longrightarrow \text { new } \mathrm{C}(\overline{\mathrm{v}}):: \mathrm{v} & \text { (R-COMP) } \\
\frac{\text { fields }(\mathrm{C})=\overline{\mathrm{C}} \overline{\mathrm{f}}}{(\text { new } \mathrm{C}(\overline{\mathrm{v}}):: \mathrm{v}) \cdot \mathrm{f}_{i} \longrightarrow \mathrm{v}_{i}} & \text { (R-FIELD) } \\
\frac{\operatorname{mbody}(\mathrm{m}, \mathrm{C})=\left(\overline{\mathrm{x}}, \mathrm{e}_{0}\right)}{(\text { new } \mathrm{C}(\overline{\mathrm{v}}):: \mathrm{v}) \cdot \mathrm{m}(\overline{\mathrm{u}}) \longrightarrow[\overline{\mathrm{x}} \leftarrow \overline{\mathrm{u}}, \text { this } \leftarrow \text { new } \mathrm{C}(\overline{\mathrm{v}}):: \mathrm{v}] \mathrm{e}_{0}} & \text { (R-INVK) } \\
\frac{\operatorname{mbody}(\mathrm{m}, \mathrm{C})=\bullet}{(\text { new } \mathrm{C}(\overline{\mathrm{v}}):: \mathrm{v}) \cdot \mathrm{m}(\overline{\mathrm{u}}) \longrightarrow \mathrm{v} \cdot \mathrm{m}(\overline{\mathrm{u}})} &
\end{array}
$$

Congruence rules

$$
\begin{array}{cc}
\frac{\mathrm{e} \longrightarrow \mathrm{e}^{\prime}}{\mathrm{e} . \mathrm{f} \longrightarrow \mathrm{e}^{\prime} \cdot \mathrm{f}} & \frac{\mathrm{e} \longrightarrow \mathrm{e}^{\prime}}{\mathrm{e} \cdot \mathrm{m}(\overline{\mathrm{e}}) \longrightarrow \mathrm{e}^{\prime} \cdot \mathrm{m}(\overline{\mathrm{e}})} \\
\frac{\mathrm{e}_{i} \longrightarrow \mathrm{e}_{i}^{\prime}}{\mathrm{v}_{0} \cdot \mathrm{m}\left(\overline{\mathrm{v}}, \mathrm{e}_{i}, \overline{\mathrm{e}}\right) \longrightarrow \mathrm{v}_{0} \cdot \mathrm{m}\left(\overline{\mathrm{v}}, \mathrm{e}_{i}^{\prime}, \overline{\mathrm{e}}\right)} & \frac{\mathrm{e}_{i} \longrightarrow \mathrm{e}_{i}^{\prime}}{\text { new } \mathrm{C}\left(\overline{\mathrm{v}}, \mathrm{e}_{i}, \overline{\mathrm{e}}\right) \longrightarrow \text { new } \mathrm{C}\left(\overline{\mathrm{v}}, \mathrm{e}_{i}^{\prime}, \overline{\mathrm{e}}\right)} \\
\frac{\mathrm{e}_{2} \longrightarrow \mathrm{e}_{2}^{\prime}}{\mathrm{e}_{1} \longleftarrow \mathrm{e}_{2} \longrightarrow \mathrm{e}_{1} \longleftarrow \mathrm{e}_{2}^{\prime}} & \frac{\mathrm{e}_{1} \longrightarrow \mathrm{e}_{1}^{\prime}}{\mathrm{e}_{1} \longleftarrow \mathrm{v} \longrightarrow \mathrm{e}_{1}^{\prime} \longleftarrow \mathrm{v}}
\end{array}
$$

Fig. 6. Semantics of IFJ

\subsection{Operational semantics}

The operational semantics, shown in Figure 6, is defined by the reduction relation e $\longrightarrow$ $\mathrm{e}^{\prime}$, read "e reduces to $\mathrm{e}^{\prime}$ in one step". The standard reflexive and transitive closure of $\longrightarrow$ defines the reduction relation in many steps. We adopt a deterministic call-byvalue semantics, analogous to the call-by-value strategy of FJ [25]. The congruence rules formalize how operators (method invocation, object creation, object completion and field selection) are reduced only when all their subexpressions are reduced to values (call-by-value).

The expression $[\overline{\mathrm{x}} \leftarrow \overline{\mathrm{u}}$, this $\leftarrow$ new $\mathrm{C}(\overline{\mathrm{v}}):: \mathrm{v}] \mathrm{e}$ denotes the expression obtained from e by replacing $\mathrm{x}_{1}$ with $\mathrm{v}_{1}, \ldots, \mathrm{x}_{n}$ with $\mathrm{v}_{n}$ and this with new $\mathrm{C}(\overline{\mathrm{v}}):: \mathrm{v}$. A runtime object is represented by a list of standard FJ objects, new $\mathrm{C}(\overline{\mathrm{v}})$; in order to treat composed objects and standard objects uniformly, we represent a standard object with a list of only one element, new $\mathrm{C}(\overline{\mathrm{v}}):: \varepsilon$. The main idea of the semantics of method 
invocation is to search for the method definition in the (class of the) head of the list using the mbody lookup function. If this is found, rule (R-INVK), then the method body is executed; otherwise, rule (R-DINVK), the search continues on the next element of the list (of course, in a well-typed program, this search will succeed eventually).

Now the following question arises: What object do we substitute for this in the method body? This is a crucial issue in order to perform field selection and further method invocations correctly (and avoid the program getting stuck). Method and field selections in a method body expect to deal with an object of the class where the method (or field) is defined (or a subclass). Thus, it is sensible to substitute this with the sublist whose head new $\mathrm{C}(\overline{\mathrm{v}})$ is such that $\operatorname{mbody}(\mathrm{m}, \mathrm{C})$ is defined. This is also consistent with the concept of consultation. Thus, the list implements the scope of this inside a method body: the scope is restricted to the visibility provided by the class where the method is defined. Note that this solves also possible ambiguities due to name clashes, even for methods hidden by subsumption [26]. Suppose we have an incomplete class $A$ that requires a method $m$ and defines a method $n$; an instance of $A$ can be completed with an object that provides $m$, say an object of class $B$ that also defines a method $n$, possibly with a different signature. When we invoke $m$ on the completed object, we actually execute the definition of $m$ in $B$; if this method then invokes $n$, the definition of $n$ in $B$ will be executed, since the scope of this is actually restricted to $B$; this is consistent with the typing that has checked the invocation of $n$ in B.m using the signature of B.n.

\section{Properties}

The language IFJ enjoys the type safety property, thus no errors "message-not-understood" can occur at run-time. In this section, we sketch this proof by showing the main steps that are related to object composition. Namely, in the formal proofs, we explicitly deal with crucial points involving typing and semantics rules for incomplete objects, while we omit the parts that are similar or unchanged with respect to the corresponding proofs in FJ.

The following key lemmas state some crucial properties about the signatures of classes $\mathrm{C}_{1}, \ldots, \mathrm{C}_{n}$ when considering values of the shape

$$
\mathrm{v}=\text { new } \mathrm{C}_{1}\left(\overline{\mathrm{v}}_{1}\right):: \ldots:: \text { new } \mathrm{C}_{n}\left(\overline{\mathrm{v}}_{n}\right):: \varepsilon
$$

Indeed, these properties are proved for the expression

$$
\mathrm{e}=\text { new } \mathrm{C}_{1}\left(\overline{\mathrm{v}}_{1}\right) \longleftrightarrow \ldots \longleftarrow \text { new } \mathrm{C}_{n}\left(\overline{\mathrm{v}}_{n}\right)
$$

using typing and semantic rules. Then, the properties are inherited by $\mathrm{v}$ since $\mathrm{v}$ can only be obtained by applying rule (R-COMP) to e (Figure 6); it is clear that the application of (R-COMP) does not affect signatures of $\mathrm{C}_{1}, \ldots, \mathrm{C}_{n}$.

Lemma 1. Let $\mathrm{v}=$ new $\mathrm{C}(\overline{\mathrm{v}}):: \mathrm{v}^{\prime}$ such that $\mathrm{v}^{\prime} \neq \varepsilon$ and $\Gamma \vdash \mathrm{v}: \mathrm{T}$ where concrete $(\mathrm{T})$. Then

1. $\Gamma \vdash \mathrm{v}^{\prime}: \mathrm{T}^{\prime}$ for some $\mathrm{T}^{\prime}$ such that concrete $\left(\mathrm{T}^{\prime}\right)$

2. If $\operatorname{sign}(\mathrm{C})=\left\langle\mathrm{S}_{1}, \mathrm{~S}_{2}\right\rangle$, for any $\mathrm{m}$ such that $\mathrm{m}: \overline{\mathrm{B}} \rightarrow \mathrm{B} \in \mathrm{S}_{2}$ then mtype $\left(\mathrm{m}, \mathrm{T}_{1}\right)=\overline{\mathrm{B}} \rightarrow \mathrm{B}$ 
Proof.

1. By (T-List) and (T-COMP).

2. By (T-COMP).

Lemma 2. If $\Gamma \vdash$ new $\mathrm{C}_{1}\left(\overline{\mathrm{v}}_{1}\right):: \ldots::$ new $\mathrm{C}_{n}\left(\overline{\mathrm{v}}_{n}\right):: \varepsilon: \mathrm{T}$ where concrete $(\mathrm{T})$, let $\operatorname{sign}\left(\mathrm{C}_{i}\right)=$ $\left\langle\mathrm{S}_{1}^{i}, \mathrm{~S}_{2}^{i}\right\rangle, 1 \leq i \leq n$, then, for any method $\mathrm{m}$ :

1. mtype $(\mathrm{m}, \mathrm{T})=\overline{\mathrm{B}} \rightarrow \mathrm{B}$ implies that there exists some $\mathrm{C}_{i}, 1 \leq i \leq n$, such that $\mathrm{m}: \overline{\mathrm{B}} \rightarrow$ $\mathrm{B} \in \mathrm{S}_{1}^{i}$

2. For any $\mathrm{C}_{i}, 1 \leq i \leq n, \mathrm{~m}: \overline{\mathrm{B}} \rightarrow \mathrm{B} \in \mathrm{S}_{1}^{i}$ implies $\operatorname{mbody}\left(\mathrm{m}, \mathrm{C}_{i}\right)=\overline{\mathrm{x}}$.e and this $: \mathrm{C}_{i}, \overline{\mathrm{x}}$ : $\overline{\mathrm{B}} \vdash \mathrm{e}: \mathrm{T}$ for some $\mathrm{T}<: \mathrm{B}$

Proof.

1. mtype $(\mathrm{m}, \mathrm{T})=\overline{\mathrm{B}} \rightarrow \mathrm{B}$ implies that $\mathrm{m}: \overline{\mathrm{B}} \rightarrow \mathrm{B} \in \mathrm{S}_{1} \cup \mathrm{S}_{2}$, where $\operatorname{sign}(\mathrm{T})=\left\langle\mathrm{S}_{1}, \mathrm{~S}_{2}\right\rangle$; then the proof follows by induction on $n$ using (T-COMP).

2. By induction on the derivation of $\operatorname{mbody}\left(\mathrm{m}, \mathrm{C}_{i}\right)$.

The following substitution property is standard and it proceeds essentially as in FJ, since incomplete classes do not introduce crucial new subtyping relations.

Lemma 3 (Substitution Lemma).

If $\Gamma, \overline{\mathrm{x}}: \overline{\mathrm{B}} \vdash \mathrm{e}: \mathrm{T}$ and $\Gamma \vdash \overline{\mathrm{e}}: \overline{\mathrm{T}}$ where $\overline{\mathrm{T}}<: \overline{\mathrm{B}}$, then $\Gamma \vdash[\overline{\mathrm{x}} \leftarrow \overline{\mathrm{e}}] \mathrm{e}: \mathrm{T}^{\prime}$ for some $\mathrm{T}^{\prime}<: \mathrm{T}$.

Proof. Straightforward induction on the derivation of $\Gamma, \overline{\mathrm{x}}: \overline{\mathrm{B}} \vdash \mathrm{e}: \mathrm{T}$.

Theorem 1 (Type Preservation).

If $\Gamma \vdash \mathrm{e}: \mathrm{T}$ and $\mathrm{e} \longrightarrow \mathrm{e}^{\prime}$ then $\Gamma \vdash \mathrm{e}^{\prime}: \mathrm{T}^{\prime}$ for some $\mathrm{T}^{\prime}<: \mathrm{T}$.

Proof. By induction on a derivation of $\mathrm{e} \longrightarrow \mathrm{e}^{\prime}$. The only interesting cases are:

- (R-COMP): By (T-COMP) and (T-LisT), the type is preserved after reduction.

- (R-INVK): By (T-INVK) and Lemma 2-2, using Substitution Lemma 3.

- (R-DINVK): By Lemma 1.

Theorem 2 (Progress). Let e be a closed expression. If $\vdash$ e : $\mathrm{T}$ for some $\mathrm{T}$, then either $\mathrm{e}$ is a value or $\mathrm{e} \longrightarrow \mathrm{e}^{\prime}$ for some $\mathrm{e}^{\prime}$.

Proof. By induction on $\vdash \mathrm{e}: \mathrm{T}$; the crucial case is:

- (T-INVK): the method invocation can be reduced either by (R-INVK) or by (R-DINVK); Lemma $2-1$ is the key argument to guarantee that the search for the method body will eventually succeed (i.e., (R-INVK) will be applied after some applications of (R-DINVK)).

Theorems 1 and 2 show how type safety of $\mathrm{FJ}$ is preserved when adding incomplete objects with our approach, i.e., any well-typed IFJ program cannot get stuck. 


\section{Dealing with imperative features}

Our language is based on FJ that abstracts the functional core of Java; then, also our approach has a functional flavor, i.e., it does not consider side effects and imperative features. Thus, it might not be clear what happens to the object state and identity during object composition, in an imperative setting.

In this section we want to show how the present approach can be adapted to an imperative version of FJ (e.g., using heaps and object identifiers similarly to [7]), based on our run-time representation of objects.

The key point is that, when objects are composed, the resulting object consists of a list of sub-objects; in particular these sub-objects are not modified. Thus, the states of the objects within an object composition never change and the object composition produces a brand new object.

Consider the (R-COMP) rule in Figure 6:

$$
\text { new } \mathrm{C}(\overline{\mathrm{v}}):: \varepsilon \longleftrightarrow \mathrm{v} \longrightarrow \text { new } \mathrm{C}(\overline{\mathrm{v}}):: \mathrm{v}
$$

The new object contains the sub-objects without changing them (note that the removal of $:: \varepsilon$ from new $\mathrm{C}(\overline{\mathrm{v}}):: \varepsilon$ is due only to our uniform representation for complete and incomplete objects and, obviously, has nothing to do with the state of the object itself).

Each object composition creates a brand new object and all the sub-objects are actually shared (for instance, in an imperative model each object composition gets a new object identifier); in particular, the code employing object composition in Listing 1 clarifies this point: the (same) complete object deleg is used for completing all the three incomplete objects. Actually we could have also written the code as follows:

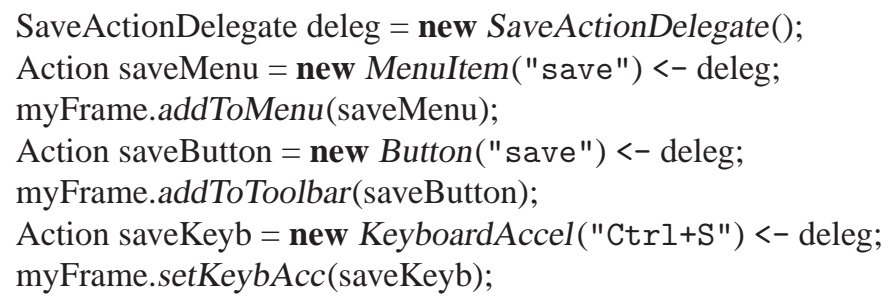

Thus, modifying the internal state of deleg will assure that all the actions are updated too. For instance, if the SaveActionDelegate had logging features, we could enable them and disable them during the execution of our program, and all the actions resulting from the object compositions will use logging consistently.

This shows that objects are not only instances of classes (possibly incomplete classes), but they are also prototypes that can be used, via the object composition, to create new objects at run-time, while ensuring statically that the composition is type safe. We then can use incomplete and complete objects as our re-usable building blocks to assemble at run-time, on the fly, brand new objects.

Furthermore, this mechanism will assure that there will not be problems when an object is pointed to by references in different parts of the program. Actually, if we modified the objects directly during the object composition, what would happen to the existing references? Surely they would all refer to the modified objects, but we would 
create situations where a reference of an incomplete class type would point to a complete object. This would break our discipline of not composing already complete objects with other complete objects; but, most of all, it would undermine the type safety (recall that there is no subtyping between an incomplete class and a complete class).

Finally, not modifying incomplete objects directly also makes them more re-usable especially in cases when object composition may not have to be permanent: the behavior of an object may need to evolve many times during the execution of a program and the missing methods provided during an object composition might need to be changed, e.g., because the state of the incomplete object has changed (we refer to the learning objects scenario in the Introduction). Since the original incomplete object is not modified, then it can be re-used smoothly in many object compositions during the evolution of a program.

\section{Ongoing extensions}

The core language IFJ is rather compact and minimal, since we focus on proving how Java basic aspects and type safety are preserved when adding incomplete objects and object composition using our approach. In this section we sketch some further developments, that are quite interesting for enhancing programming flexibility, and we provide hints on how to deal with them (the full technical treatment of these further language constructs is the subject of future work).

A first extension could be permitting to redefine a method provided during object composition. In this case, the incomplete object can provide the implementation of a method $m$ relying on the implementation of $m$ that must be provided (at run-time) during object composition. This is similar to standard method overriding, but it takes place dynamically and at object level.

We then introduce another syntax for such redefined methods (in order to distinguish them by standard method overriding): redef $\mathrm{C} m(\overline{\mathrm{C}} \overline{\mathrm{x}})\{$ return e; $\}$. In order to access the implementation of this method provided by the object used in object composition, we can use the keyword next from within a redef method (e.g., next.m()), in order to distinguish it from super 4 . Thus, with next, one can access the "next" object in the object list. Just like this, next is a variable that will be implicitly bound in redef methods.

With method redefinition we could easily implement a stream library, as sketched in Listing 2: the specific stream specializations rely on the methods provided during object completion (using next) and specialize them.

A further enhancement would be to provide delegation, instead of simple consultation. When implementing delegation, we need substitute for this the whole object (the whole list, not only the part that was scanned when searching for the method definition) and this will require representing an object as a pair of lists (the second one is used as a backup and represents the entire object, while the first one is the part examined when searching for the method definition).

\footnotetext{
${ }^{4}$ Actually in FJ super is not considered; however, if introduced, it could coexist with next.
} 


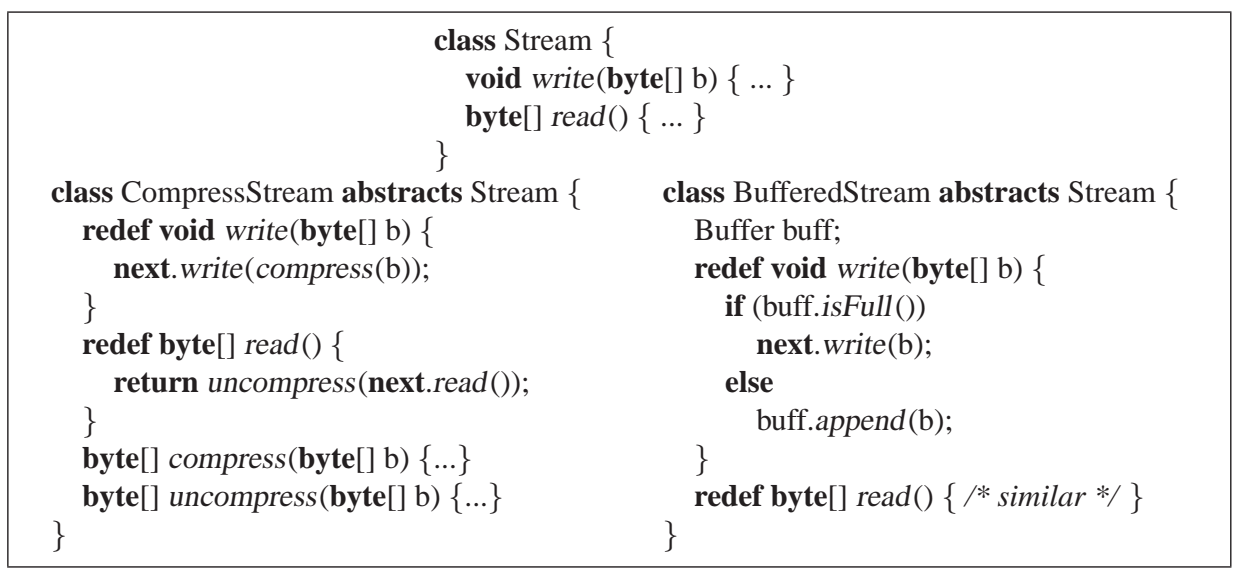

Listing 2: The implementation of streams using redefined methods

It would also be quite useful to be able to compose two incomplete objects obtaining another incomplete object; this would permit having an incomplete object that can be completed in more than one step. For instance, consider the CompressStream in Listing 2: we could make the two compress and uncompress methods incomplete, so that we will be able to complete the CompressStream object with a stream object (providing the read and write functionalities that will be redefined) and a compressor object (implementing the actual compression algorithm). This way, a CompressStream can be reused independently from the stream and from the compression algorithm.

Finally, we could extend the subtyping relation also to incomplete classes (and thus incomplete objects); in particular, an incomplete object $o_{1}$ has a subtype of an incomplete object $o_{2}$ if $o_{1}$ has no more abstract methods than $o_{2}$ (thus, it has less requirements), while it must have exactly the same redefined methods (see also $[6,5]$ ).

\section{Conclusions and related works}

In this paper we presented Incomplete Featherweight Java (IFJ), an extension of Featherweight Java with incomplete objects, i.e., objects that require some missing methods which can be provided at run-time by composition with another (complete) object, combining the static type discipline class-based features with the flexibility of object-based ones. The language IFJ enjoys the type safety property, thus no error "message-notunderstood" can occur at run-time. The implementation of incomplete objects in Java is currently under development.

Incomplete objects seem to be a useful language construct to deal with the problem of dynamic reconfiguration of mobile code [6], where incomplete software components can be sent over a network to be completed with local components. Furthermore, in the context of service oriented programming and web services, mechanisms enabling service composition and reconfiguration, based on types, could be implemented through incomplete objects. In this direction, we plan to investigate how our approach to object 
composition can be exploited in calculi that incorporate session types in an objectoriented framework, such as [14].

An explicit form of incomplete objects was introduced in [8], where an extension of Lambda Calculus of Objects of [17] is presented. In this work, "labelled" types are used to collect information on the mutual dependencies among methods, enabling a safe subtyping in width. Labels are also used to implement the notion of completion which permits adding methods in an arbitrary order allowing the typing of methods that refer to methods not yet present in the object, thus supporting a form of incomplete objects. The context is the lambda calculus, while in this work we are interested in incorporating object composition into Java-like languages.

In [22] delegation is presented in the model of the language Darwin; however, this model requires some basic notions to be modified, such as method overriding. Our language, instead, proposes a conservative extension of a Java-like language (so that existing code needs not to be changed). Furthermore, in [22] the type of the parent object must be a declared class and this limits the flexibility of dynamic composition, while in our approach there is no implicit parent and missing methods can be provided by any complete object, independently from its class.

Incomplete objects can be seen as wrappers for the objects used in object composition. However, they differ from decorator-based solutions such as the language extension presented in [7]: incomplete objects provide a more general-purpose language construct and the wrappers of [7] could be actually implemented through incomplete objects.

Traits [15] are composable units containing only methods, and they were proposed as an add-on to traditional class-based inheritance in order to enhance decoupling and high cohesion of code in classes, therefore with the aim of allowing a higher degree of code reuse. Incomplete objects can be seen as a tool for rapid prototyping, that is, for adding methods on the fly to already existing objects. Traits and incomplete objects share an important feature, composition, which permits composing sets of methods "at the right level", for instance not too high in a hierarchy for traits, and "when needed" for incomplete objects. The main difference is that traits are a compile-time feature, while incomplete objects are composed at run-time. An issue to pursue as a further research may be the use of incomplete objects as an exploratory tool to design traits: experiments made at run-time without modifying a class hierarchy might give indications on where to put a method in a new version of the hierarchy.

There are some relations between aspects [13] and our incomplete objects. Both are used to combine features taken from different sources. In the aspect case, the main idea is to factorize into aspects some cross-cutting functions (such as logging services or concurrency primitives) that are needed globally by a library, instead of duplicating and scattering them into the business code. In our case, we consider objects as building blocks that can be used to combine features on the fly, in order to obtain and experiment with multi-function objects whenever it is desired. In a sense, the role of incomplete objects is orthogonal to the one of aspects, because the former play a local role, while the latter a more global one.

In [2], a general model (Method Driven Model) for languages supporting object composition is proposed: this is based on the design of classes in an aspect-oriented 
style. The authors do not formalize their model within a calculus, but it is possible to see that the main feature of a language based on this model would be to compose dynamically the overall behavior of an object from the multiple "aspects" that abstract the variant behavior, as discussed in [3]. The main difference between their proposal and ours is that for them the run-time behavior is codified in aspects, whereas we internalize it in Java by exploiting partial classes and object composition.

The language gbeta [16] supports a mechanism called "object metamorphosis", which is a mechanism to specialize dynamically an existing object, by applying to it a class as a constraint in such a way the object becomes an instance of that class. The main difference between the gbeta specializing objects and our incomplete objects is that the former maintain the object identity, while the latter are used to create dynamically new objects which are not instances of any classes present in the program. Both proposals are proved type-safe, but a more direct comparison is not straightforward, as the type system of gbeta exploits concepts such as virtual classes which are not present in a Java-like setting like ours. It is important to remark that one of our main design decision was that our extension must integrate seamlessly in a Java-like language as a conservative extension.

Acknowledgments. We thank the anonymous referees for comments which helped us to improve the paper.

\section{References}

1. C. Anderson, F. Barbanera, M. Dezani-Ciancaglini, and S. Drossopoulou. Can Addresses be Types? (a case study: Objects with Delegation). In WOOD'03, volume 82(8) of ENTCS, pages 1-22. Elsevier, 2003.

2. C. Babu and D. Janakiram. Method Driven Model: A Unified Model for an Object Composition Language. ACM SIGPLAN Notices, 39(8):61-71, 2004.

3. C. Babu, W. Jaques, and D. Janakiram. DynOCoLa: Enabling Dynamic Composition of Object Behaviour. In Proc. 2nd International Workshop on "Reflection, AOP and MetaData for Software Evolution (RAM-SE)” at ECOOP 2005, 2005.

4. L. Bettini, V. Bono, and S. Likavec. Safe and Flexible Objects with Subtyping. Journal of Object Technology, 10(4):5-29, December 2005. Special Issue: OOPS Track at SAC 2005.

5. L. Bettini, V. Bono, and B. Venneri. Subtyping-Inheritance Conflicts: The Mobile Mixin Case. In Proc. Third IFIP International Conference on Theoretical Computer Science (TCS 2004). Kluwer Academic Publishers, 2004.

6. L. Bettini, V. Bono, and B. Venneri. MoMi: a calculus for mobile mixins. Acta Informatica, 42(2-3):143-190, 2005.

7. L. Bettini, S. Capecchi, and E. Giachino. Featherweight Wrap Java. In Proc. of SAC 2007, Special Track on Object-Oriented Programming Languages and Systems (OOPS), pages 1094-1100. ACM Press, 2007.

8. V. Bono, M. Bugliesi, M. Dezani-Ciancaglini, and L. Liquori. A Subtyping for extensible, incomplete objects. Fundamenta Informaticae, 38(4):325-364, 1999.

9. T. Boyle. Design principles for authoring dynamic, reusable learning objects. Australian Journal of Educational Technology, 19(1):46-58., 2003.

10. G. Bracha. The Programming Language Jigsaw: Mixins, Modularity and Multiple Inheritance. PhD thesis, University of Utah, 1992. 
11. G. Bracha, M. Odersky, D. Stoutamire, and P. Wadler. Making the future safe for the past: Adding genericity to the Java programming language. In OOPSLA '98 Conference Proceedings, volume 33(10) of ACM SIGPLAN Notices, pages 183-200, Oct. 1998.

12. C. Chambers. Object-Oriented Multi-Methods in Cecil. In Proc. of ECOOP, volume 615 of LNCS, pages 33-56. Springer, 1992.

13. D. Crawford. Communications of the ACM archive - Special Issue on Aspect-Oriented Programming, volume 44. ACM New York, 2001.

14. M. Dezani-Ciancaglini, D. Mostrous, N. Yoshida, and S. Drossopoulou. Session Types for Object-Oriented Languages. In Proc. of ECOOP, volume 4067 of LNCS, pages 328-352. Springer, 2006.

15. S. Ducasse, O. Nierstrasz, N. Schärli, R. Wuyts, and A. Black. Traits: A mechanism for finegrained reuse. ACM Transactions on Programming Languages and Systems, 28(2):331-388, 2006.

16. E. Ernst. gbeta - a Language with Virtual Attributes, Block Structure, and Propagating, Dynamic Inheritance. PhD thesis, Department of Computer Science, University of Aarhus, Århus, Denmark, 1999. Url: http://www.daimi.au.dk/ eernst/gbeta/.

17. K. Fisher, F. Honsell, and J. C. Mitchell. A lambda-calculus of objects and method specialization. Nordic J. of Computing, 1(1):3-37, 1994.

18. K. Fisher and J. C. Mitchell. A Delegation-based Object Calculus with Subtyping. In Proc. of FCT, volume 965 of LNCS, pages 42-61. Springer, 1995.

19. E. Gamma, R. Helm, R. Johnson, and J. Vlissides. Design Patterns: Elements of Reusable Object-Oriented Software. Addison-Wesley, 1995.

20. A. Goldberg and D. Robson. Smalltalk 80: The Language. Addison-Wesley, 1989.

21. A. Igarashi, B. Pierce, and P. Wadler. Featherweight Java: a minimal core calculus for Java and GJ. ACM Transactions on Programming Languages and Systems, 23(3):396-450, 2001.

22. G. Kniesel. Type-Safe Delegation for Run-Time Component Adaptation. In Proc. of ECOOP 99, volume 1628 of $L N C S$, pages 351-366. Springer, 1999.

23. H. Lieberman. Using prototypical objects to implement shared behavior in object oriented systems. ACM SIGPLAN Notices, 21(11):214-214, 1986.

24. D. R. Musser and A. A. Stepanov. Generic Programming. In Symbolic and Algebraic Computation. International Symposium (ISSAC '88), volume 358 of LNCS, pages 13-25. Springer, 1989.

25. B. C. Pierce. Types and Programming Languages. The MIT Press, Cambridge, MA, 2002.

26. J. Riecke and C. Stone. Privacy via Subsumption. Information and Computation, (172):2-28, 2002. 3rd special issue of Theory and Practice of Object-Oriented Systems (TAPOS).

27. A. Taivalsaari. On the notion of inheritance. ACM Computing Surveys, 28(3):438-479, Sept. 1996.

28. D. Ungar and R. B. Smith. Self: The power of simplicity. ACM SIGPLAN Notices, 22(12):227-242, 1987.

29. J. Viega, B. Tutt, and R. Behrends. Automated Delegation is a Viable Alternative to Multiple Inheritance in Class Based Languages. Technical Report CS-98-03, UVa Computer Science, 1998. 